\title{
X-RAY IMAGES OF PKS1209-52 AND ITS CENTRAL COMPACT X-RAY SOURCE
}

Yutaka Matsui

Dept. of Physics \& Astronomy, Northwestern Univ.

Evanston, IL 60201 USA

Knox S. Long

Center for Astrophysical Sciences, Johns Hopkins Univ.

Baltimore, MD 21218 USA

Ian R. Tuohy

Mount Stromlo and Siding Spring Observatories, Australian National Univ., ACT 2606, Canberra, Australia

Abstract: A complete X-ray image of the SNR PKS1209-52 (G296.5+10.0) was obtained with the IPC and HRI on the Einstein observatory. The remnant has a she11-1ike X-ray morphology much like its appearance at radio wavelengths, while a compact $X$-ray source is clearly detected near the center of the remnant. The flux observed from the $X-r a y$ nebula $F_{(0.1-4.5 \mathrm{keV})}$ is $8 \times 10^{-11}$ ergs $\mathrm{cm}^{-2} \mathrm{~s}^{-1}$, which corresponds to a luminosity $\mathrm{L}_{(0.1-4.5 \mathrm{keV})}=8 \times 10^{35}$ ergs $\mathrm{s}^{-1}$ for a distance of $2 \mathrm{kpc}$. Applying a simple she 11 model to the $\mathrm{X}$-ray emission distribution, we derived an ambient interstellar medium $\mathrm{n}_{0}=0.08 \mathrm{H}$ atoms $\mathrm{cm}^{-3}$, total $\mathrm{x}$-ray emitting plasma mass $150 \mathrm{Mo}$, and thermal energy $1.2 \mathrm{x}$ $10^{50}$ ergs. The flux from the compact $X$-ray source $\mathrm{F}(0.15-4.5 \mathrm{keV})$ is $-2 \times 10^{-12}$ ergs $\mathrm{cm}^{-2} \mathrm{~s}^{-1}$. There are no obvious optical counterparts brighter than $\mathrm{m}_{\mathrm{v}}-22$ within the $3.3 "$ radius HRI error circle. If the object is a hot neutron star, the HRI/IPC count rate ratio implies a surface temperature of $1.6 \times 10^{6} \mathrm{~K}$ for $\mathrm{N}_{\mathrm{H}}=3.2 \times 10^{21} \mathrm{~cm}^{-2}$.

Introduction: The SNR PKS1209-52 was first detected as a soft X-ray source with $H E A O-1$ by Tuohy et al. (1979). They fitted the X-ray pulse height distribution with a model spectrum of cosmic abundance plasma at collisional equilibrium (Raymond and Smith 1977) with a temperature of $1.9 \times 10^{6} \mathrm{~K}$ and a column density of $3.2 \times 10^{21} \mathrm{H}$ atoms $\mathrm{cm}^{-2}$. Applying a simplified Sedov solution, they derived an ambient ISM density $\sim 0.4$ $\mathrm{cm}^{-3}$, an initial supernova energy $7 \times 10^{50} \mathrm{ergs}$, and an age of 20000 years for a distance of $2 \mathrm{kpc}$. Recently Kellett et al. (1987) observed the bright eastern portion of the remnant using the EXOSAT Observatory and obtained a soft $X$-ray image and a spectrum. The $X$-ray spectrum was fitted with the Raymond and Smith thin hot plasma model with solar abundances. The best fit was obtained for a temperature of $1.7 \times 10^{6} \mathrm{~K}$ and a column density of $1.4 \times 10^{21}$ atoms $\mathrm{cm}^{-2}$. Thus PKS1209-52 is the SNR whose $\mathrm{X}$-ray emitting plasma indicates the lowest characteristic temperature ever observed.

The distance to PKS1209:52 is poorly known since it has been estimated only from the radio $\Sigma$ - D relation. The distance estimates are in the range from $1.1 \mathrm{kpc}$ (Milne 1979) to $1.9 \mathrm{kpc}$ (Caswell and 
Lerche 1979). On the other hand, Mills (1983) has suggested that the distance to galactic SNRs is more accurately given by $\mathrm{d}_{\mathrm{kpc}}=$ $\left(1280 / \mathrm{S}_{408} \mathrm{MHz}\right)^{1 / 2}$, where $\mathrm{S}_{408} \mathrm{MHz}$ is the total flux at $408 \mathrm{MHz}$ in $\mathrm{Jy}$. This gives a distance of $3.9 \mathrm{kpc}$ for PKS1209-52. We adopt a distance of $2 \mathrm{kpc}$ for the analysis following.

Einstein Observations and Results: Observations of PKS1209-52 were carried out with the Imaging Proportional Counter (IPC) and the High Resolution Imager (HRI) on the Einstein Observatory (Giacconi et al. 1979). Eight IPC exposures were obtained to map the total extent of the SNR; exposure times ranged from 1570 to $2880 \mathrm{sec}$. In addition, a follow-up $5930 \mathrm{sec}$. observation of the SE limb was obtained. Using only the central $36^{\prime} \times 36^{\prime}$ of each field to avoid severe vignetting, the individual images were merged into a single map shown in Figure 1 . The remnant appears as a heavily fragmented but roughly circular ring at $\mathrm{X}$-ray wavelengths. In addition, there is a compact $\mathrm{X}$-ray source near the center of the ring. The point-like nature of this source was subsequently confirmed with the HRI. The HRI image (Figure 2) was obtained on January 22,1981 with $3930 \mathrm{sec}$. exposure. The compact source is located at $\alpha=12^{\mathrm{h}} 07^{\mathrm{m}} 23 . \mathrm{s} 50, \delta=-52^{\circ} 09^{\prime} 49^{\prime \prime}$ (1950) with an error radius of 3.3 " (908 confidence). The IPC count rate was $0.085 \pm$ $0.005 \mathrm{cts} \mathrm{s}^{-1}$ on July 11,1979 and the HRI rate was $0.015 \pm 0.002 \mathrm{cts}$ $s^{-1}$. The IPC count rate corresponds to an energy flux $-2 \times 10^{-12}$ ergs $\mathrm{cm}^{-2} \mathrm{~s}^{-1}$ between 0.15 and $4.5 \mathrm{keV}$. The $X$-ray image is similar to the radio map at $5 \mathrm{GHz}$ obtained by Milne and Dickel (1975). The only noticeable difference between the $X$-ray and radio images is in the NW, where there is very weak $X$-ray emission despite the moderately strong radio emission. As there is a correlation between $\mathrm{X}$-ray and radio surface brightnesses in SNRs (Matsui et al. (1984); Berkhuijsen (1986) ), the X-ray appearance of PKS1209-52 is mostly intrinsic. The outer edge of the $X$-ray and radio emission and the optical filaments in the north roughly follow a circle of radius $36^{\prime}$ centered at $\alpha=12^{\mathrm{h}} 06^{\mathrm{m}}$ $47 .{ }^{\circ} 3, \delta=-52^{\circ} 12^{\prime} 29^{\prime \prime}(1950)$, which we identify as the geometrical center of the remnant.

Spectral analysis of our IPC data without reprocessing is difficult due to the complex spatial gain variation of the detector. So we adopt the plasma model and the interstellar absorption derived from the $H E A O-1$ data. For this model the IPC count rate of 3.61 cts $\mathrm{s}^{-1}$ for the remnant (excluding the point source) corresponds to an energy flux of $7.8 \times 10^{-11}$ ergs $\mathrm{cm}^{-2} \mathrm{~s}^{-1}$ between 0.1 and $4.5 \mathrm{keV}$, and an intrinsic $X$-ray luminosity $\left.L_{(0.1-4.5} \mathrm{keV}\right)=7.8 \times 10^{35}$ ergs $\mathrm{s}^{-1}$. For the adopted temperature and $\mathrm{N}_{\mathrm{H}}$, apparent $\mathrm{X}$-ray emissivity $\varepsilon_{\mathbf{x}}=5.0 \mathrm{x}$ $10^{-14}$ IPC cts $\mathrm{cm}^{5} \mathrm{~s}^{-1}$ (see Figure 11 of Leahy et al. 1985). Taking the geometrical center as the center of the SNR, we obtained the radial X-ray surface brightness distribution of the entire remnant. For a uniform density shell, the observed maximum limb-brightening of 3.7 corresponds to a ratio of shell thickness to outer radius $\Delta R / R=0.16$. Since the maximum surface brightness, $1.23 \times 10^{-3} \mathrm{cts} \operatorname{arcmin}^{-2} \mathrm{~s}^{-1}$, is at $R-\Delta R$ $=29^{\prime}$, we have $R=35^{\prime}$ and $\Delta R=6^{\prime}$. Then we have a density in the shell $\mathrm{n}_{e}=0.23 \mathrm{~cm}^{-3}$ and an ambient medium density $\mathrm{n}_{0}=0.08 \mathrm{H} \mathrm{cm}^{-3}$. The mass of the plasma producing the total $\mathrm{X}$-ray emission is $150 \mathrm{Mo}$ and the thermal energy content $E_{t h}=1.2 \times 10^{50}$ ergs. 

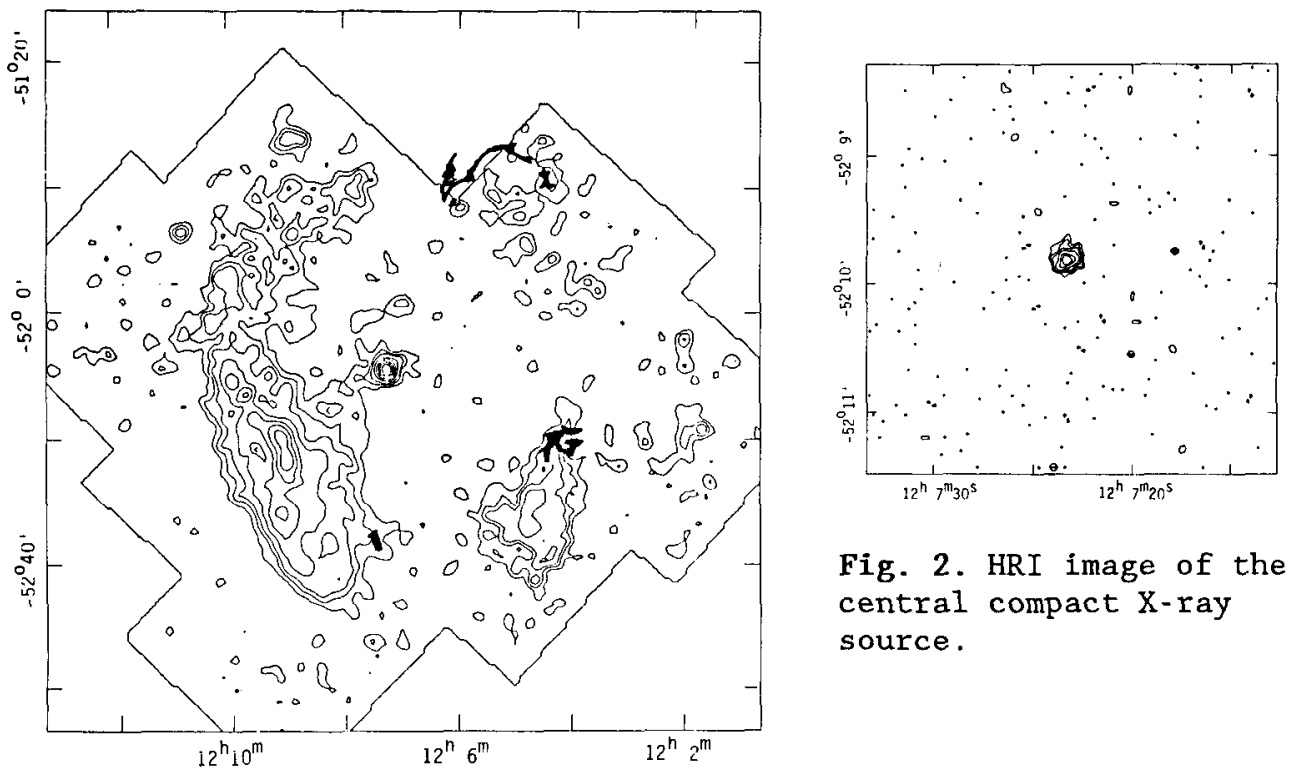

Fig. 2. HRI image of the central compact $\mathrm{X}$-ray source.

Fig. 1. Smoothed IPC image of PKS1209-52. Contours are 0.6, $1.1,1.6,2.6,3.6,4.6,5.6$, and $6.6 \times 10^{-3} \mathrm{cts} \operatorname{arcmin}^{-2}$ $\mathrm{s}^{-1}$. FWHM of the pont spread function is $-2.8^{\prime}$. The positions of optical nebulosity are indicated by dark areas.

The Compact X-ray Source near the Center of PKS1209-52: The HRI error circle of the point $X$-ray source is shown in Figure 3 , overlaid on the ESO blue plate. Only one star (designated A) is visible within the error circle, 2.2" to the north of the $X$-ray centroid. We obtained a low resolution $(-10 \AA)$ spectrum of this star using the Anglo-Australian Telescope on June 18, 1981. The spectrum of star A corresponds to that of a normal $\mathrm{K}$ subdwarf with $\mathrm{V}=17.5, \mathrm{U}-\mathrm{B}=1.4, \mathrm{~B}-\mathrm{V}=1.2$, and $\mathrm{V}-\mathrm{R}-$ 0.5 . An identification of star $A$ with the point $X$-ray source is not plausible in view of the ratio $\log \left(F_{X} / F_{V}\right)=1.0$, which is at least 3 orders of magnitude greater than that expected from a $\mathrm{K}$ star (Vaiana et al. 1981). A recent radio map of PKS1209-52 obtained at a frequency of $843 \mathrm{MHz}$ with 40 " beam sets an upper limit of $4 \mathrm{mJy}$ for a source at the position (Roger 1986). A pulsar search made by Manchester, D'Amico, and Tuohy (1985) yielded an upper limit of $1 \mathrm{mJy}$ at $1.4 \mathrm{GHz}$. For typical pulsar parameters (e.g. Tuohy et al. 1983), the limit implies a radio luminosity between $10^{8}-10^{9} \mathrm{~Hz}$ of $<1.2 \times 10^{28} \mathrm{ergs} \mathrm{s}^{-1}$. This value is a factor of 3 below that of the weakest radio pulsar (PSR1509-58) associated with a SNR (see Table 1 in Tuohy et al. 1983).

Helfand and Becker (1984) estimated a probability of 0.04 for finding an $X$-ray source of the observed intensity in an area of the SNR. However, this probability reduces to only 0.0006 for finding an $\mathrm{X}$-ray source within $6.1^{\prime}$ of the geometrical center. For an assumed age of $20000 \mathrm{y}$, the transverse velocity required to move $6.1^{\prime}$ is $-170 \mathrm{~km} \mathrm{~s}^{-1}$, which is not at all unreasonable compared with measured pulsar 
velocities of $20-500 \mathrm{~km} \mathrm{~s}^{-1}$ (e.g. Helfand, Chanan, and Novick 1980). A hot neutron star emitting blackbody radiation is a viable possibility for the X-ray source. Here we adopt the model of a neutron star with a stellar radius $\mathrm{R}_{\mathrm{o}}=16.1 \mathrm{~km}$ and a mass $\mathrm{M}=1.31$ Mo proposed by Pandharipande, Pines, and Smith (1976). The gravitational redshift factor at the surface of the neutron star $1+z=\left(1-2 \mathrm{GM} / \mathrm{c}^{2} \mathrm{R}_{0}\right)^{-1 / 2}=1.15$. We calculated the expected IPC and HRI count rates from a hot neutron star and found that the observed HRI/IPC count rate ratio is consistent with a surface temperature $\mathrm{T}_{\mathrm{O}}=1.6 \times 10^{6} \mathrm{~K}$ if $\mathrm{N}_{\mathrm{H}}=3.2 \mathrm{x}$ $10^{21} \mathrm{~cm}^{-2}$. The surface temperature derived above is close to the bestfit blackbody temperature of $1.8 \times 10^{6} \mathrm{~K}$ derived by using the EXOSAT Observatory (Kellett et al. 1987). Such a temperature is commensurate with the surface temperature of a neutron star predicted by Nomoto and Tsuruta (1986) even for an age of $-2 \times 10^{4}$ years.

\section{References}

Berkhuijsen, E.M. 1986, Astr. Astrophys., 166, 257.

Caswell, J.L. and Lerche, I. 1979, M.N.R.A.S., 187, 201.

Ciacconi, R. et al. 1979, Ap. J., 230, 540.

Helfand, D.J. and Becker, R.H. 1984, Nature, 307, 215.

Helfand, D.J., Chanan, G.A., and Novick, R. 1980, Nature, 283, 337.

Kellett, B.J. et a1. 1987, M.N.R.A.S., 225, 199.

Leahy, D.A., Venkatesan, D., Long, K.S., and Naranan, S. 1985, Ap. J., $294,183$.

Manchester, R.N., D'Amico, N., and Tuohy, I.R. 1985, M.N.R.A.S., 212, 975.

Matsui, Y., Long, K.S., Dickel, J.R., and Greisen, E.R. 1984, Ap. J., $287,295$.

Mills, B.Y. 1983, Supernova Remnants and their X-ray Emission, ed. J. Danziger and P. Gorenstein (Dordrecht: Reide1), p. 551.

Milne, D.K. 1979, Aust. J. Phys., 32, 83.

Milne, D.K. and Dickel, J.R. 1975, Aust. J. Phys., 28, 209.

Nomoto, K. and Tsuruta, S. 1986, Ap. J. (Letters), 305, L19.

Pandharipande, V.R., Pines, D., and Smith, R.A. 1976, Ap. J., 208, 550. Raymond, J.C., and Smith, B.W. 1977, Ap. J. Suppl., 35, 419.

Roger, R.S. 1986, Private Communication.

Tuohy, I.R., Garmire, G.P., Manchester, R.N., and Dopita, M.A. 1983, Ap. J., 268, 778 .

Tuohy, I.R. et al. 1979, Ap. J. (Letters), 230, L27.

Vaiana, G.S. et al. 1981, Ap. J., 245, 163. 